\title{
Caso Clínico: Aplicación de Fibrina Rica en Factores de Crecimiento en el Tratamiento de Osteonecrosis de los Maxilares por Bisfosfonatos
}

\section{Clinical Case: Application of Rich Fibrin in Growth Factors in the Treatment of Osteonecrosis of the Maxillary by Bisphosphonates}

Recibido: 2018/06/15. Aceptado: 2018/08/02. Publicado: 2018/09/01

\section{Jorge León Batallas ${ }^{1}$ \\ Patricio Unda Jaramillo ${ }^{2}$ \\ Andres Ortiz Onofre ${ }^{3}$}

1 Universidad San Francisco De Quito, Colegio De Ciencias De La Salud, Escuela De Odontología, Clínica de Odontología, Campus Cumbayá, oficina CO 106, casilla postal 17-200-841. Quito - Ecuador.

Correo electrónico: jalbcmf@hotmail.com

2 Universidad San Francisco De Quito, Colegio De Ciencias De La Salud, Escuela De Odontología, Clínica de Odontología, Campus Cumbayá, oficina CO 106, casilla postal 17-200-841. Quito - Ecuador.

Correo electrónico: patundaja@hotmail.com

3 Universidad San Francisco De Quito, Colegio De Ciencias De La Salud, Escuela De Odontología, Clínica de Odontología, Campus Cumbayá, oficina CO 106, casilla postal 17-200-841. Quito - Ecuador.

Correo electrónico: andres_nanopaulo@outlook.es 


\section{Resumen}

Este trabajo trata del manejo de la osteonecrosis de los maxilares relacionada a los bisfosfonatos y el uso de la fibrina rica en plaquetas y leucocitos como agente de estimulación, aceleración de la cicatrización de tejidos y la regeneración ósea. Aquí se describe un caso clínico con el objetivo de demostrar un tipo de técnica que permite el cierre total de la exposición ósea moderada secundaria a la osteonecrosis obteniendo resultados óptimos a los 6 meses, tomando en cuenta que puede existir la alternativa de la utilización de la fibrina rica en plaquetas, leucocitos y factores de crecimiento, comprobando ser una terapia nueva, eficaz en el tratamiento de estas lesiones.

Palabras claves: osteonecrosis, bisfosfonatos, factores de crecimiento, L-PRF

\section{Abstract}

This paper deals with the management of osteonecrosis of the jaws related to bisphosphonates and the use of fibrin rich in platelets and leukocytes as stimulation agent, acceleration of tissue healing and bone regeneration. Here we describe a clinical case with the objective of demonstrating a type of technique that allows the total closure of moderate bone exposure secondary to osteonecrosis obtaining optimal results after 6 months, taking into account that there may be an alternative to the use of fibrin rich in platelets, leukocytes and growth factors, proving to be a new, effective therapy in the treatment of these injuries.

Keywords: osteonecrosis, bisphosphonates, growth factors, L-PRF 


\section{Introducción}

La osteonecrosis de los maxilares relacionada con el uso de los bisfosfonatos (ONMBF) es una lesión caracterizada por la exposición de hueso necrótico que persiste por más de 8 semanas, acompañada de una zona ulcerada con inflamación, supuración, dolor y parestesia, por lo general tras un procedimiento quirúrgico dentoalveolar menor, como la exodoncia, la cirugía periodontal, cirugía Implantológica, etc $\mathbf{1 , 2}$.

El mecanismo de acción del bisfosfonato (BFF) se da por la inhibición de la reabsorción ósea a partir de la supresión de la actividad osteoclástica ya que actúa directamente sobre la célula principal que inicia la remodelación del hueso produciendo en ella la muerte o apoptosis celular $\mathbf{1 , 2 , 1 5}$.

Estos medicamentos utilizados básicamente para el tratamiento de la osteoporosis, osteopenia, metástasis óseas a partir de carcinomas como el de mama o próstata actúan de manera óptima con acciones beneficiosas, sin embargo, también hay efectos adversos por la acción de estos fármacos ${ }^{4}$.

El plasma rico en fibrina y leucocitos (L-PRF) es un concentrado plaquetario de segunda generación descrito por Choukroun en el año 2001. Su constitución básica tiene tres componentes: plasma, leucocitos y plaquetas a partir de la sangre autóloga sin la presencia de aditivos, estructuralmente este concentrado es un cítoesqueleto muy rígido en donde los gránulos plaquetarios liberan factores de crecimiento que son mediadores biológicos naturales que ejercen varios efectos sobre los procesos de reparo y regeneración ósea ya que acelera la angiogénesis provocando eventos celulares como la migración, la proliferación y diferenciación celular, ya que sus bondades han sido aplicadas en la actualidad para el tratamiento de la cirugía implantológica avanzada que incluye el manejo de los injertos óseos, sin embargo, recientes estudios, han mostrado grandes beneficios en el tratamiento de la osteonecrosis de los maxilares por bisfosfonatos 5,6 .

\section{Caso clínico}

Paciente sexo femenino de 70 años de edad con antecedente de osteoporosis postmenopáusica, bajo tratamiento con ácido ibandrónico (BONOSE) 150mg VO una vez al mes durante 5 años. Llega al Servicio de Cirugía Oral y Maxilofacial del Hospital HE-1 de las Fuerzas Armadas con sintomatología dolorosa. Al examen clínico, se evidencia herida expuesta al medio bucal a nivel de reborde alveolar de región posterior mandibular derecha, con supuración y sangrado constante, a causa de una extracción

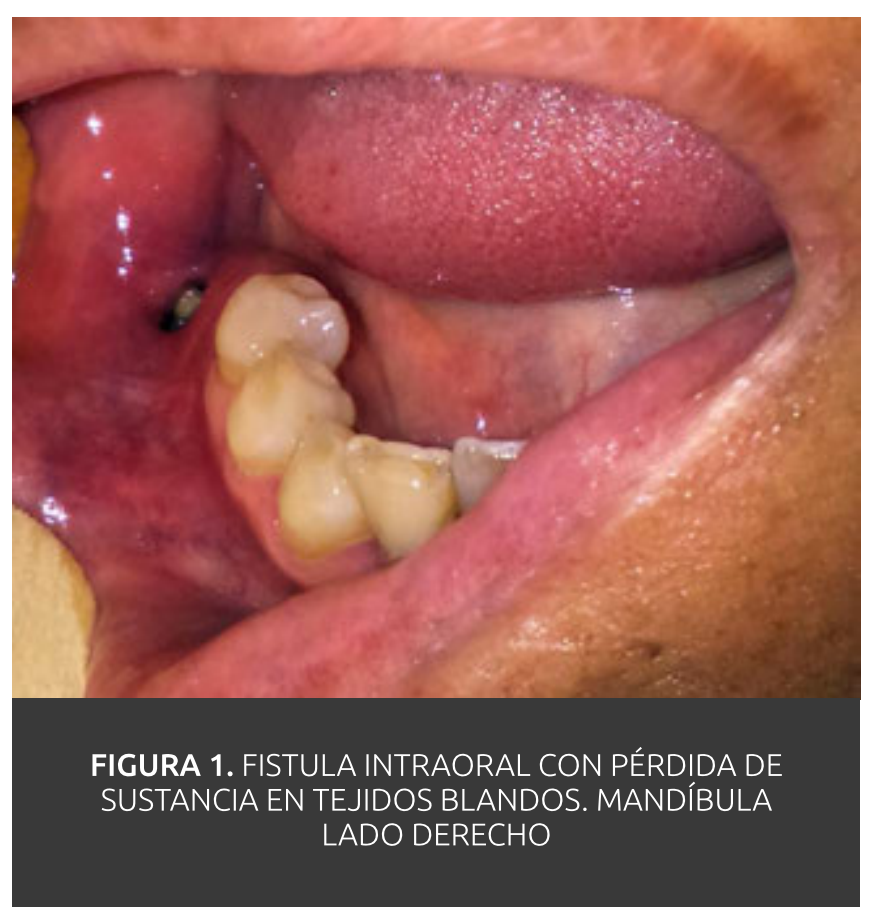

dental hace 4 años (Figura 1).

El diagnóstico clínico y tomográfico fue una osteonecrosis de los maxilares relacionada con el uso de los bisfosfonatos (ONMBF) estadio IIB basado en la clasificación de R. Marx; se descontinuó 1 año la terapia con ibandronato antes de la propuesta quirúrgica, que consistió en la limpieza quirúrgico con la eliminación de hueso necrótico y la utilización de la fibrina rica en plaquetas, leucocitos y factores de crecimiento (Figura 2). 


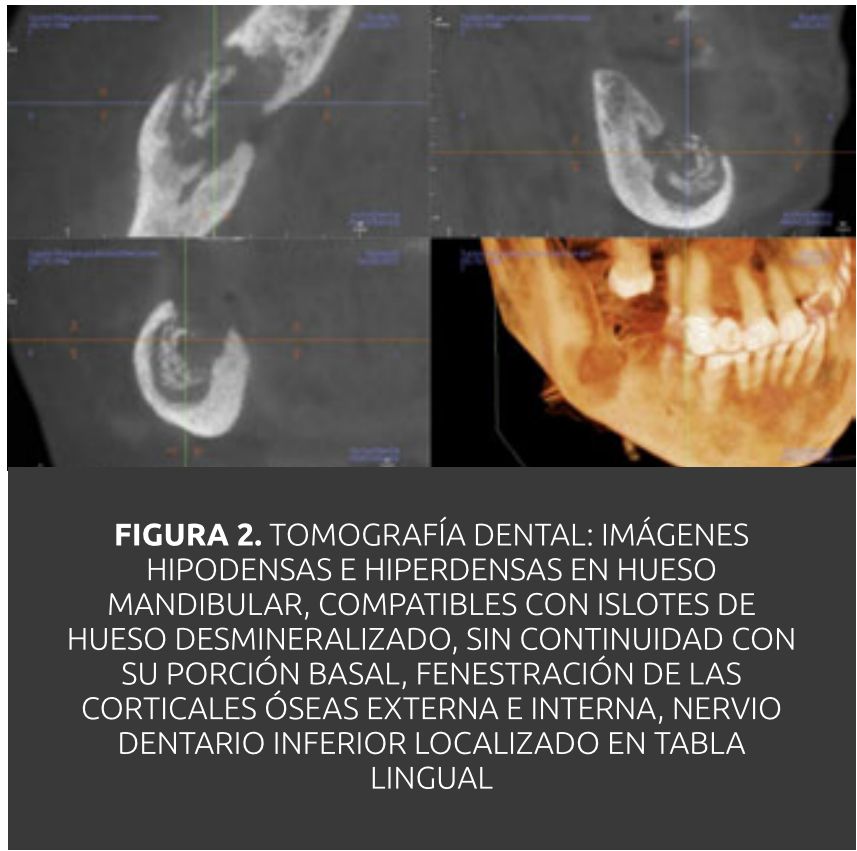

La paciente fue medicada preoperatoriamente con analgésicos (paracetamol 1gr e ibuprofeno $600 \mathrm{mg}$ ), antibióticos (amoxicilina + ácido clavulánico $625 \mathrm{mg}$ ) y el uso de colutorios de gluconato de clorhexidina $0,12 \%$ haciendo enjuagues bucales tres veces al día después de cada comida. En la tomografía dental se pudo valorar la extensión de la osteonecrosis (Figura 2), el protocolo quirúrgico incluyó la resección del tejido necrótico, remoción del tejido de granulación y la secuestrectomía con retiro de los

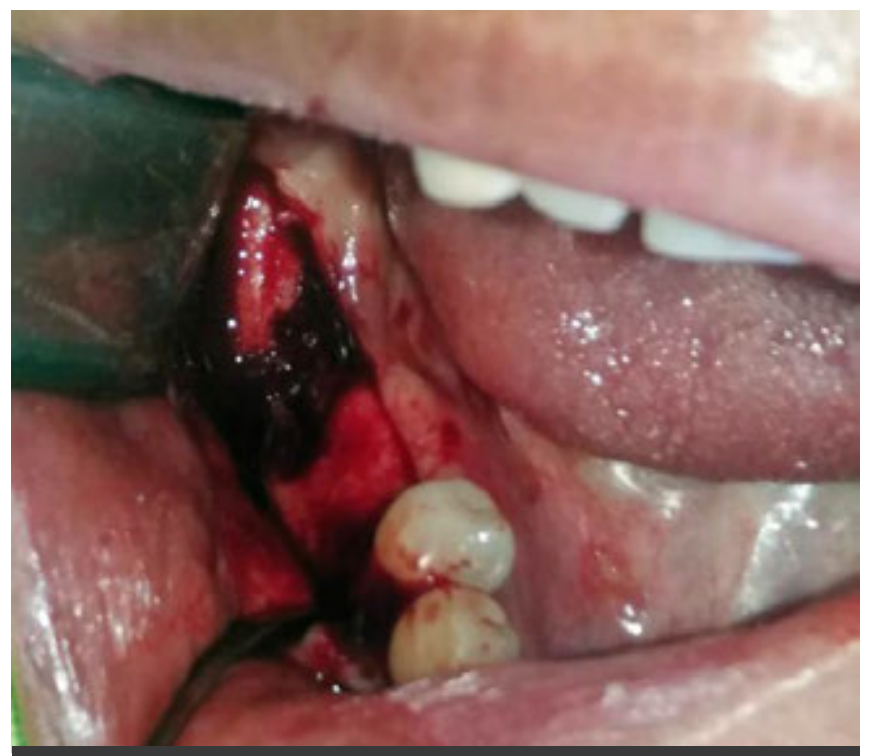

FIGURA 3. ELIMINACIÓN DE TEJIDO ÓSEO NECRÓTICO Y PRESERVACIÓN DEL NERVIO DENTARIO INFERIOR segmentos en forma manual hasta la presencia del sangrado ósea alrededor de todas las paredes del hueso (Figura 3).

Después, la herida fue irrigada con solución de cloruro de sodio al 0,9\% más 2 ampollas de clindamicina $600 \mathrm{mg}$, eliminando cuerpos extraños y coágulos. Luego se colocó un coágulo y 2 membranas de fibrina rica en plaquetas y leucocitos (L-PRF) alrededor de las paredes óseas y dentro de la cavidad (Figura 4), este proceso se basó en un centrifugado de 2.700 rpm a 12 minutos con la toma de muestra de sangre en tubos de $10 \mathrm{~mL}$. Finalmente, se realizó la síntesis con sutura reabsorbible 3-0 (poliglactina 910), los cuales fueron retirados a los 8 días postquirúrgicos. Se manejó con antibioterapia adicional de levofloxacina 500mg, 1 tableta diaria durante 15 días.

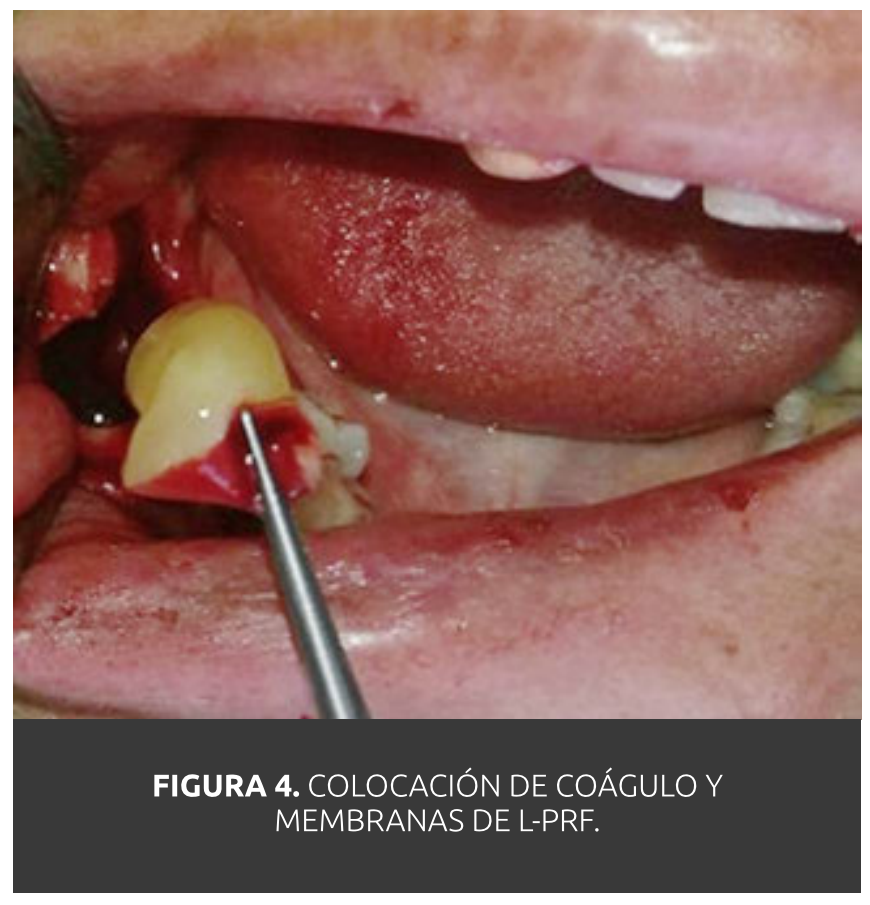

El procedimiento quirúrgico consistió en resección de tejido óseo necrótico más colocación de fibrina rica en plaquetas y leucocitos (L-PRF). Se realizó seguimiento del primer mes (Figura 5) y control de 6 meses 


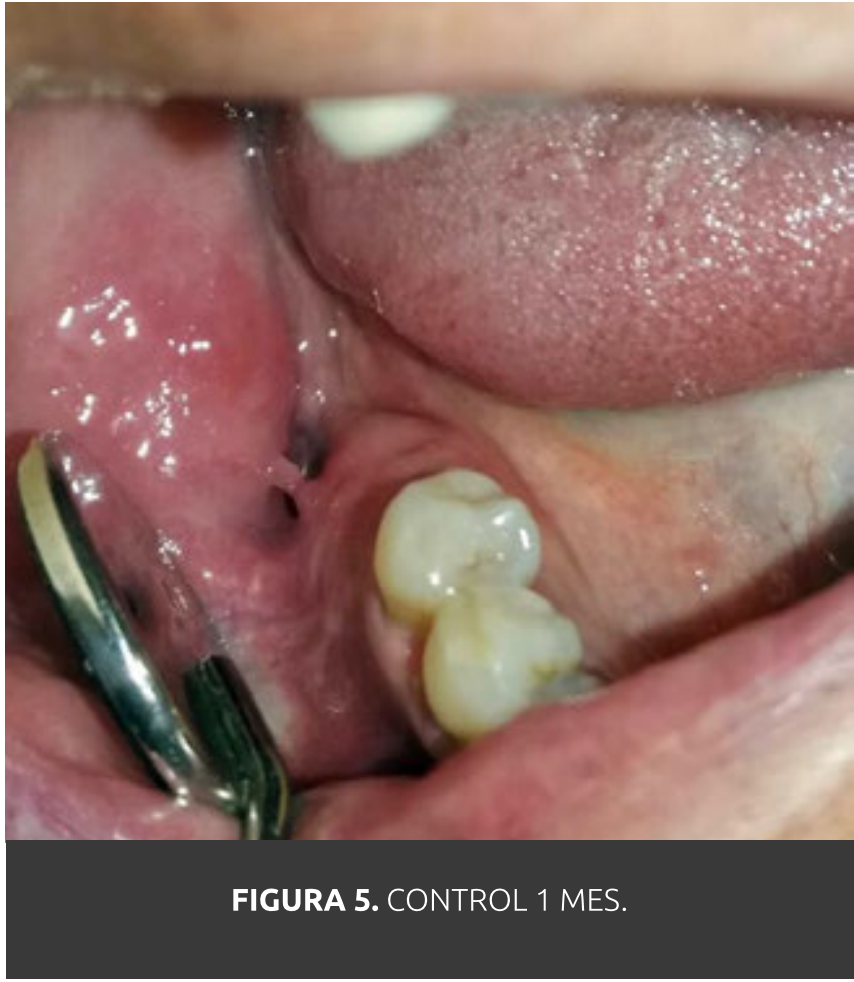

(Figura 6) mostrándose asintomática y clínicamente la herida en proceso de cicatrización con revestimiento mucoso total de las paredes óseas.

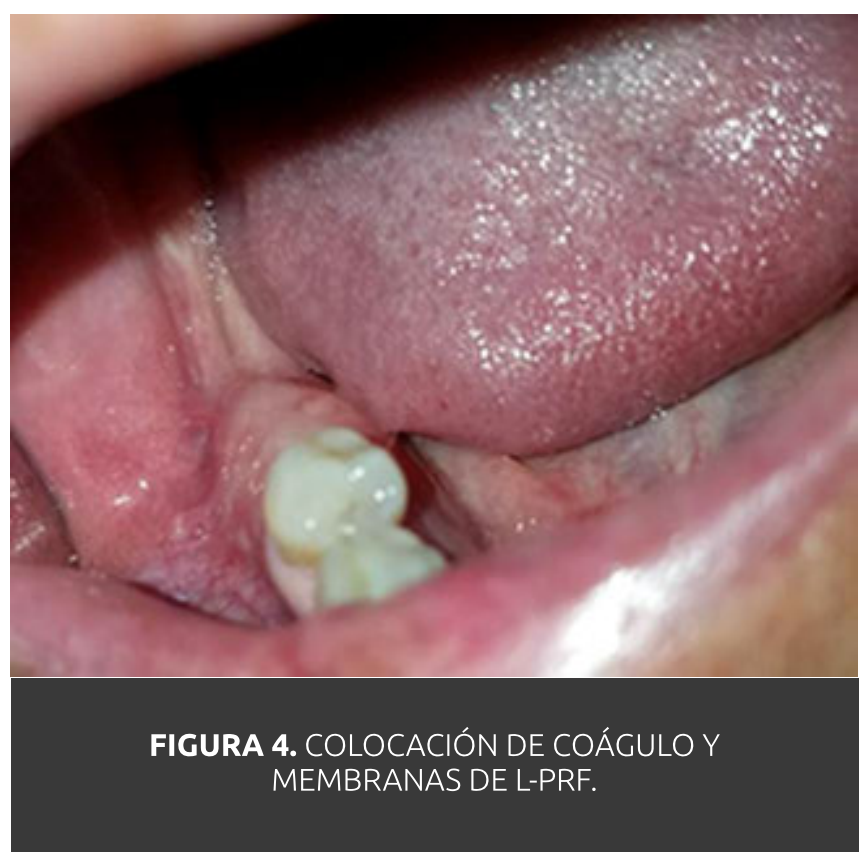

\section{Discusión}

En el tratamiento del ONMBF se requiere aliviar el dolor, reducir la infección y estabilizar la progresión de la enfermedad con el cierre de la exposición ósea. Algunos casos publicados, que pueden estar asociados con la inhibición del LRANK, se han manejado de forma conservadora, utilizando antibióticos a largo plazo más enjuagues locales. Estos tratamientos se ha informado que no han tenido éxito en menos del $60 \%$ de los $\operatorname{casos}^{1,7}$.

Nuestro objetivo fue evaluar la viabilidad del uso L-PRF para el tratamiento de ONMBF. Dado que actualmente no existe un tratamiento universalmente aceptado, la aplicación de este tratamiento fue importante ya que, es el primer caso reportado en nuestro país que muestra resultados prometedores.

A pesar de las nuevas recomendaciones de la American Association of Oral and Maxillofacial Surgeons (AAOMS), la tasa de éxito del tratamiento de antibioterapia y el uso de colutorios, como manejo conservador está entre el $20-50 \%$, lo que es significativamente menor que la tasa de éxito de más del $85 \%$ reportados en la terapia quirúrgica $\mathbf{8 , 3}^{\mathbf{3}}$.

Algunos estudios anteriores han informado que el tratamiento combinado de eliminación del hueso necrótico y curetaje de la superficie más la colocación de fibrina rica en plaquetas leucocitos y factores de crecimiento, parece ser prometedor para el tratamiento de ONMBF 9,10.

Los BFF pueden liberarse rápidamente en la cresta alveolar y en un medio bucal ácido, dando lugar a concentraciones altas después de procedimientos quirúrgicos invasivos en los pacientes medicados con estos fármacos que podrían afectar a los osteoclastos, osteoblastos, células endoteliales, fibroblastos y queratinocitos 11,12 . 
Este método incorpora el uso de concentrado de L-PRF se caracteriza por la ausencia de los aditivos de productos químicos, lo que permite que el proceso de coagulación sea natural, el LPRF no se disuelve rápidamente después del uso, preservando la estructura de su matriz de fibrina 1.

La concentración autóloga de plaquetas humanas que se obtienen por la centrifugación de sangre, produce un alta contenido de factores de crecimiento proteícos que son secretados activamente por las plaquetas. La técnica ha sido sugerida para diversos propósitos, incluyendo la estimulación de la producción de colágeno, del crecimiento vascular, la disminución de procesos inflamatorios y el aumento en la cicatrización de las heridas ${ }^{13,15}$.

Pripatnanont y col., encontraron que el PRF estaba compuesto de densas redes de fibrina, con plaquetas activadas. Estas fibrinas densas proporcionan una matriz, como especie de andamio natural para el almacenamiento y el acoplamiento de las células del tejido y la estimulación de la angiogénesis. Además, las plaquetas proporcionan y mantienen la liberación lenta y continua de factores de crecimiento (PDGF, TGF beta 1-2, VEGF) involucrados en la cascada angiogénica ${ }^{1}$.

Estudios recientes han descrito la capacidad de LPRF para aumentar la proliferación celular en osteoblastos y fibroblastos del ligamento periodontal y pulpa mediante la regulación positiva de osteoprotegerina y fosfatasa alcalina $6,3,8$

Dell Fabbro y col., en una revisión sistemática, sugirió que el uso de concentraciones plaquetarias como complemento al procedimiento de cirugía oral, puede tener un efecto beneficioso para la prevención, ocurrencia o recurrencia postquirúrgica en pacientes bajo tratamiento con bifosfonatos $\mathbf{4 , 6 , 1 1}$.
Soydan - Uckan usaron dos capas de membrana de L-PRF después de la extirpación quirúrgica de ONMBF, en un seguimiento de 4 meses había un cierre completo de la exposición del hueso ${ }^{\mathbf{8 , 1 2}}$.

Kim y col., evaluaron a 34 pacientes en el que se combinó L-PRF con resección quirúrgica. Las evaluaciones se realizaron después de 4 meses, con 26 pacientes (77\%) presentando resolución completa, 6 (18\%) con retraso en la cicatrización, y 2 (6\%) no mostrando ninguna resolución ${ }^{\mathbf{1 , 1 1}}$.

Varios estudios también han sugerido el papel positivo de los leucocitos en los concentrados de plaquetas, tales como la actividad antimicrobiana, la regulación inmunológica, y la capacidad de producir grandes cantidades de VEGF. En estudios anteriores, indican que los enfoques conservadores con L-PRF fueron menos efectivos en la enfermedad más avanzada y puede ser necesaria una intervención prematura para evitar que la enfermedad empeore $\mathbf{1 2 , 1 0 , 1 4}$.

\section{Conclusiones}

Este estudio describe la aplicación del L-PRF como agente de regeneración ósea en la BIONJ, dando resultados positivos en la cicatrización de heridas en su seguimiento a corto de plazo de 6 meses.

El uso de plasma rico en factores de crecimiento para la regeneración tisular garantizaría la disponibilidad de factores de crecimiento y citoquinas en las fases inicial y crítica de la cicatrización de tejidos.

La eliminación del tejido necrótico y el legrado de la superficie combinado con terapia antibiótica, mejora los síntomas relacionados con BIONJ y ayudan a cicatrizar el hueso.

Las membranas L-PRF son una alternativa fácil, barata y rápida para el tratamiento en el cierre de la exposición ósea por BIONJ ya que promueven la curación gingival y actúa como una membrana de barrera entre el hueso alveolar y la cavidad oral. 


\section{Referencias bibliográficas}

1. Kim, J., Kim, S., Kim, M. Leucocyte-rich and platelet-rich fibrin for the treatment ofbisphosphonate-related osteonecrosis of the jaw: aprospective feasibility study. British journal of oral and maxillofacial surgery. 2014; 52(9): p. 854-859.

2. Fung. P, Bedogni. G, Bedogni. A, Petrie. A, Porter. S, Campisi. G, Bagan. J, Fusco. V, Saia. G, Acham. S, Musto. P, Petrucci.M, Diz. P, Colella. G, Mignogna.M, Pentenero. M, Arduino. P, Lodi G, Maiorana. C, Manfredi. M. Time to onset of bisphosphonaterelated osteonecrosis of the jaws: a multicentre retrospective cohort study. 2016 December.

3. Maluf. G, Caldas. R, Silva. P. The use of leukocyte-and platelet-rich fibrin (LPRF) in the treatment of medication related osteonecrosis of the jaws (MRONJ). Journal of Oral and Maxillofacial Surgery. 2016 June 6.

4. Del Fabbro M. et al. Autologous platelet concentrates for bisphosphonate-related osteonecrosis of the jaw treatment and prevention. A systematic review of the literature. European Journal of Cancer. 2014; 15(1): p. 62-74.

5. G. Maluf, M. Correia. Surgery Combined with LPRF in Denosumab Osteonecrosis of the Jaw: Case Report. Brazilian Dental Journal. 2016; 27(3): p. 353-358.

6. N. Kaibuchi, T. Iwata et al. Multipotent mesenchymal stromal cell sheet therapy for bisphosphonate-related osteonecrosis of the jaw in a rat model. Acta Biomaterialia. 2016; 15(42): p. 400-410.
7. Orión. A, Salgado. A, Arriba. L. Nuevas tendencias en regeneración tisular: fibrina rica en plaquetas y leucocitos. Revista Española de Cirugia Oral y Maxilofacial. 2016 Marzo 17; 295.

8. S. Soydan, S. Uckan. Management of Bisphosphonate-Related Osteonecrosis of the Jaw with a Platelet-Rich Fibrin Membrane: Technical Report. Journal of Oral and Maxillofacial Surgery. 2013; 72(2): p. 1-5.

9. A. Cieslik et cols. Autologous platelets and leukocytes can improve healing of infected high-energy soft tissue injury. Transfusion and Apheresis Science. 2009; 41(1): p. 9-12.

10. S. Nørholt, J. Hartlev. Surgical treatment of osteonecrosis of the jaw with the use of surgical treatment of osteonecrosis of the jaw with the use of 15 patients. Internacional Journal Oral and Maxillofacial Surgery. 2016; 45(10): p. 1256-1260.

11. D, Dohan et al. Choukroun's platelet-rich fibrin (PRF) stimulates in vitro proliferation and differentiation of human oral bone mesenchymal stem cell in a dosedependent way. Archives of Oral Biology. 2010; 55(3): p. 185-194.

12. S. Ghanaati et cols. Advanced platelet-rich fibrin: a new concept for cell-based tissue engineering by means of inflammatory cells. Journal of Oral Implantology. 2014; 40(6): p. 679-689.

13. Shahrokh C. Bagheri, R. Bryan Bell. Current Therapy in Oral and Maxillofacial Surgery St. Louis: Elsevier Inc.; 2012. 
14. Rupel. K, Ottaviani. G, Gobbo. M, Contardo. L, Tirelli. G, Vescovi. P, Di Lenarda. R, Biasotto. M. A systematic review of therapeutical approaches in bisphosphonates-related osteonecrosis of the jaw (BRONJ). Oral Oncology. 2014 August; 50: p. 1049-1057.

15. Pathak. H, Mohanty. S, Aadithya. B, Dabas. J. Treatment of oral mucosal lesions by scalpel excision and PRF membrane grafting: a review of 26 sites. Journal of Oral and Maxillofacial Surgery. 2016 March; 13. 